\title{
The investigation of polymer-siRNA nanoparticle for gene therapy of gastric cancer in vitro
}

This article was published in the following Dove Press journal:

International Journal of Nanomedicine

4 March 2010

Number of times this article has been viewed

Ying $W u^{\prime}$

Weiwei Wang ${ }^{2}$

Yinting Chen'

Kaihong Huang'

Xintao Shuai ${ }^{2}$

Qikui Chen'

Xuexian $\mathrm{Li}^{\prime}$

Guoda Lian'

'Department of Gastroenterology, The Second Affiliated Hospital of Sun Yat-Sen University, Guangzhou, China; ${ }^{2}$ BME Center, School of Chemistry and Chemical Engineering, Sun Yat-Sen University, Guangzhou, China
Correspondence: Kaihong Huang Department of Gastroenterology, The Second Affiliated Hospital of Sun Yat-Sen University, Guangzhou 510120, China

Tel +862081332489

Fax +862081332244

Email huangkaih@2Icn.com

Xintao Shuai

BME Center, School of Chemistry and Chemical Engineering, Sun

Yat-Sen University, Guangzhou 510275 , China

Tel $+862084 I \mid 2105$

Fax +862084 II 2245

Email shuaixt@mail.sysu.edu.cn
Abstract: Small interfering RNA (siRNA) molecules have significant therapeutic promise for the genetic treatment of cancer. To overcome instability and low transfection efficiency, polyethylene glycol-polyethyleneimine (PEG-PEI) was synthesized and investigated as a non-viral carrier of siRNA targeting CD44v6 in gastric carcinoma cells. The size, surface charge using zeta potential, and morphology via scanning electron microscopy (SEM) of PEG-PEI/siRNA nanoparticles was characterized, and their cytotoxicity, transfection efficiency, and interaction with SGC7901 human gastric carcinoma cells was evaluated. The transfection efficiency of PEG-PEI/siRNA nanocomplexes was dependant on the charge ratio between amino groups of PEG-PEI and phosphate groups of siRNA (N/P) values, which reflected the molar ratio of PEG-PEI to siRNA during complex formation. The transfection efficiency of PEG-PEI/siRNA at N/P 15 was 72.53\% $\pm 2.38 \%$, which was higher than that observed using Lipofectamine 2000 and PEI as delivery carriers. Cytotoxicity of PEG-PEI was determined by MTT (3-[4,5-dimethylthiazol-2-yl]-2,5-diphenyltetrazolium bromide) assay and was obviously lower than that of PEI. Moreover, when N/P was below 15, PEG-PEI/siRNA was less toxic than Lipofectamine 2000/siRNA. RT-PCR (real time polymerase chain reaction) and Western blot analyses of CD44v6 expression demonstrated the gene silencing effect of PEG-PEI/siRNA at N/P 15. These data indicate that PEG-PEI may be a promising non-viral carrier for altering gene expression in the treatment of gastric cancer with many advantages, such as relatively high gene transfection efficiency and low cytotoxicity.

Keywords: siRNA, PEG-PEI, nanoparticles, CD44v6 gene, gastric carcinoma cells

\section{Introduction}

CD44 is a multifunctional cell adhesion molecule and a cell surface receptor of hyaluronan. In addition, it is involved in extracellular matrix binding, cell migration, and lymphocyte homing. ${ }^{1}$ There are a number of isoforms of CD44 resulting from alternative splicing, which includes the following: CD44s (the standard form), CD44E (the epithelial form), and CD44v (the variant isoform). ${ }^{2} \mathrm{CD} 44 \mathrm{v}$ isoforms, especially CD44v6, play an important role in tumor progression and metastasis of gastric cancer. CD44v6 expression is significantly higher in gastric cancer patients with liver metastasis. ${ }^{3}$ Moreover, the expression of CD44v6 is an independent risk factor for lymph node metastasis. ${ }^{4}$ Therefore, suppressing CD44v6 expression might postpone or inhibit the invasion and metastasis of gastric cancer.

RNA interference (RNAi) is an effective technology for gene silencing in gene therapy. Since the discovery of its mechanism, small interference RNA (siRNA) has been used for assaying gene function ${ }^{5,6}$ and potential therapeutics. ${ }^{7,8} \mathrm{~A}$ growing number

submit your manuscript $\mid$ www.dovepress.com 
of studies have shown its therapeutic promise in treating various diseases, including cancer. ${ }^{9}$ However, siRNA is rapidly degraded and the uptake by target cells and tissues is often low. ${ }^{10}$ Therefore, development of an efficient siRNA delivery system is desperately demanded.

Carrier systems are essential to deliver siRNA efficiently and overcome both extracellular and intracellular barriers. ${ }^{11}$ Liposome and polycationic polymer-based non-viral systems are common methods of delivering siRNA in vitro. As compared with viral delivery, these methods induce less immunogenicity and fewer safety issues. ${ }^{12}$ Moreover, polymer-based non-viral vectors have greater advantages than liposomes with respect to safety, convenient large-scale production, and physiological stability. The cationic polymer, polyethyleneimine (PEI) contains primary, secondary, and tertiary amines; therefore, it has the ability to complex with both DNA or siRNA, a characteristic known as the "proton sponge effect". ${ }^{13}$ Various PEI types (branched and linear) with varying molecular weights $(10,25,800 \mathrm{kDa})$ have been successfully used as non-viral gene delivery carriers in both in vitro and in vivo studies. However, PEI-based gene transfection technology may induce cell cytotoxicity, depending on the molecular weight and concentration of the polymer. ${ }^{14}$ Therefore, in the present study, we have developed a PEG-modified PEI polymer, which displays reduced cytotoxicity while maintaining considerable gene transfection efficiency. ${ }^{15-18}$ Moreover, PEG modification of PEI can increase polymer solubility, protect nanoparticles from macrophage uptake, prolong the circulation time of nanoparticles in vivo, and decrease non-specific interactions with serum protein. ${ }^{19,20}$

In this study, we synthesized a PEG-modified PEI copolymer and determined its ability to mediate the delivery of CD44v6 siRNA. PEG (2 kDa) and PEI (25 kDa) were chosen to form the copolymer; their ratio was optimized to reduce cytotoxicity and particle size. In addition, siRNA and PEG-PEI complexes were formed at different N/P ratios (theoretical charge ratio between amino groups of PEG-PEI and phosphate groups of siRNA). ${ }^{13,21}$ For low cytotoxicity and high transfection efficiency, PEG-PEI/siRNA, at the most suitable N/P ratio, was selected to transfect SGC7901 human gastric carcinoma cells.

\section{Materials and methods}

\section{Materials and reagents}

Poly(ethyleneglycol)-polyethylenimine (PEG; 2 kDa and PEI; $25 \mathrm{kDa}$, respectively) was synthesized by the School of Chemistry and Chemical Engineering at Sun Yat-Sen
University. (3-(4,5-dimethyl-thiazol-2-yl)-2,5-diphenyl tetrazolium bromide (MTT) was obtained from SigmaAldrich (St Louis, MO, USA). The PrimeScript ${ }^{\mathrm{TM}}$ RT-PCR Kit was purchased from TaKaRa Biotechnology, (Dalian, China). Cell culture medium and fetal bovine serum (FBS) were purchased from GIBCO (Carlsbad, CA, USA). The human gastric carcinoma cell line, SGC-7901, was obtained from the Institute of Biochemistry and Cell Biology at the Chinese Academy of Sciences (Shanghai, China). siRNA targeting human CD44v6, negative control siRNA, and FAM-labeled siRNA were purchased from GenePharma (Shanghai, China). Antibodies specific for human CD44v6 were purchased from Bender MedSystems ${ }^{\circledR}$ (Vienna, Austria). The following siRNA targeting sequences were used: siRNA-CD44v6, 5'CAG UGG UUU GGC AAC AGA UTT 3'; negative control siRNA, 5'GCG ACG AUC UGC CUA AGA 3'; and FAM-labeled siRNA, 5'UUC UCC GAA CGU GUC ACG UTT 3'.

\section{Cell culture}

Human gastric carcinoma SGC-7901 cells were cultured and maintained in high glucose Dulbecco's modified Eagle's medium (DMEM) supplemented with 10\% fetal bovine serum at $37^{\circ} \mathrm{C}$ in a fully humidified atmosphere of $5 \% \mathrm{CO}_{2}$.

\section{Preparation of PEG-PEI copolymers}

Methoxy N-hydroxysuccinimide polyethylene glycol (mPEG2k-NHS) was prepared as described previously. ${ }^{22}$ To synthesize PEG-PEI, $1.25 \mathrm{~g}$ of hyperbranched PEI (25 kDa, Aldrich) and $1.0 \mathrm{~g} \mathrm{mPEG-NHS} \mathrm{was} \mathrm{added} \mathrm{to} \mathrm{phosphate}$ buffered saline (PBS, pH 7.4). The solution was magnetically stirred at room temperature overnight. The resulting solution was purified by membrane dialysis (molecular weight cutoff: $7 \mathrm{kDa}$ ) in distilled water for 48 hours, and then lyophilized to obtain solid PEG-PEI. PEG-PEI was characterized by Proton Nuclear magnetic resonance (1H-NMR) in deuterium oxide.

\section{Preparation of siRNA-polymer nanocomplexes}

PEG-PEI copolymers were dissolved to yield different concentrations $(0.24-1.42 \mathrm{mg} / \mathrm{mL})$, according to various $\mathrm{N} / \mathrm{P}$ ratios. Appropriate volumes of siRNA $(20 \mu \mathrm{M})$ and PEGPEI solutions were added to deionized water after which it was gently mixed and incubated 10-15 minutes at room temperature to induce nanocomplex formation. 


\section{Zeta potential and size measurements}

Nanoparticle size was analyzed using the Zeta-Plus instrument (Brookhaven, NY, USA) at $25^{\circ} \mathrm{C}$. Zeta potentials of the nanoparticles were measured using the same instrument at a $45^{\circ}$ angle and $25^{\circ} \mathrm{C}$. The data represent the average \pm standard deviations.

\section{Scanning electron microscopic analysis}

The morphology of PEG-PEI/siRNA nanocomplexes was observed by scanning electron microscopy (SEM; Quanta 400 FEG). Nanocomplexes were deposited onto a coverslip and dried for 2 hours at room temperature. After they were gilded, they were observed in the thermal field of the electron microscope.

\section{Gel retardation assay}

Gel electrophoresis was performed to evaluate siRNA condensation. PEG-PEI/siRNA complexes were prepared at different $\mathrm{N} / \mathrm{P}$ ratios from 5 to 30 , and the complexes were loaded onto $1 \%$ agarose gels with $6 \times$ loading buffer $(1 \mu \mathrm{L})$ and $10 \times$ colloidal gold $(1 \mu \mathrm{L})$. The mixture was separated in $0.5 \times$ Tris/Borate/EDTA (TBE) buffer at $100 \mathrm{~V}$ for 25 minutes siRNA bands were visualized using an ultra violet (UV) imaging system (Uvidoc, UVItec Ltd, Cambridge, UK).

\section{MTT assay}

SGC7901 cells were grown in 96-well plates at an initial density of 9000 cells/well in $200 \mu \mathrm{L}$ medium and incubated for 24 hours prior to the addition of polymers. The cytotoxicities of PEI and PEG-PEI at different final concentrations were tested. Each concentration was analyzed in triplicate. In addition, the cytotoxicity of PEG-PEI/siRNA at different N/P ratios was also evaluated; Lipofectamine 2000 and PEI $(1 \mathrm{mg} / \mathrm{mL})$ were used as controls. Transfection was performed with $20 \mathrm{pM}$ siRNA per well to form the PEG-PEI/ siRNA complexes. After additional incubation for 48 hours, the medium was replaced with $200 \mu \mathrm{L}$ serum-free medium, and $20 \mu \mathrm{L}$ MTT solution $(5 \mathrm{mg} / \mathrm{mL})$ were added per well. After 4 hours, $100 \mu \mathrm{L}$ of DMSO was added. The plate was agitated for 10 minutes, and the absorbance of each well was recorded with an ELISA reader (Labsystem Dragon, Oy, Finland) at $492 \mathrm{~nm}$. The data from three separate experiments were calculated as a percentage of viable cells over untreated control \pm standard deviation.

\section{Transfection efficiency analysis}

SGC7901 cells were seeded into 6-well plates at a density of $4 \times 10^{4}$ cells per well before transfection. The PEG-
$\mathrm{PEI} / \mathrm{siRNA}$ complexes were prepared, and according to the N/P ratio, from 5 to 30,100 pM FAM-siRNA and copolymer solution at different concentrations were added into $500 \mu \mathrm{L}$ PBS. The PEG-PEI/siRNA complexes were gently mixed and incubated for 10-15 minutes at room temperature. The original cell culture media was replaced with $1 \mathrm{~mL}$ of fresh and complete DMEM per well. The complexes were added to the well drop by drop and then rocked gently for 5 hours at $37^{\circ} \mathrm{C}$ after which the transfection media was exchanged with fresh, complete DMEM. The cells were observed under the fluorescence microscope (Nikon, Tokyo, Japan), and images were recorded.

Sixteen hours after transfection, cells were washed with PBS and detached with $0.25 \%$ trypsin/EDTA. The cells were washed with PBS and resuspended in $500 \mu \mathrm{L}$ PBS. The transfection efficiency was evaluated by scoring the percentage of cells with green fluorescence using a FACSCalibur system (Becton Dickinson, Franklin Lakes, NJ, USA). The data were presented as mean \pm standard deviation.

\section{RT-PCR analysis}

After transfection for 48 hours, RNA was extracted using Trizol reagent (Invitrogen, Carlsbad, CA, USA). RT-PCR using the PrimeScript RT-PCR Kit was performed following the manufacturer's instructions; $2 \mu \mathrm{L}$ resulting cDNA were subjected to PCR reactions using specific primers. Negative control (without cDNA) was used to detect possible contamination. PCR reaction was as follows: 30 cycles including $94^{\circ} \mathrm{C}$ for 30 seconds, $56^{\circ} \mathrm{C}$ for 30 seconds, and $72^{\circ} \mathrm{C}$ for 30 seconds and 1 cycle at $72^{\circ} \mathrm{C}$ for 7 minutes. PCR products were analyzed on $2 \%$ agarose gels. Sequences of the CD44v6 primers were as follows: forward, $5^{\prime}$-GGC AAC TCC TAG TAG TAC AAC-3' and reverse, 5'-CAG CTG TCC CTG TTG TCG AAT-3'. Sequences of the glyceraldehyde 3-phosphate dehydrogenase (GADPH) primers were as follows: forward, $5^{\prime}$-TTC GAC AGT CAG CCG GAT CTT C- $3^{\prime}$ and reverse, $5^{\prime}$-CTT CTC CAT GGT GGT GAA GAC G-3'.

\section{Western blot analysis}

After transfection for 72 hours, total protein was extracted in RIPA buffer (Beyotime, Shanghai, China) with protease inhibitors (PMSF). Protein concentrations were measured using the BCA-100 Protein Quantitative Analysis Kit (Shenergy Biocolor, Shanghai, China). Equal amounts of total proteins were separated in $10 \%$ sodium dodecyl sulfate (SDS) polyacrylamide gel and transferred to polyvinylidene 
difluoride (PVDF) membranes. The membranes were blocked in 5\% nonfat dry milk in Tris-buffered saline tween-20 (TBST) for 2 hours at room temperature and incubated with mouse anti-CD44v6 antibodies (1:1000, Bender, Austria) overnight at $4^{\circ} \mathrm{C}$. After three washes with TBST, membranes were incubated with anti-mouse IgG-horseradish-peroxidase (1:5000, Jackson, MI, USA) for 1 hour at room temperature. Enhanced chemiluminescence detection reagent was used, and the protein bands were visualized after exposition with $\mathrm{x}$-ray film. GADPH expression was used as the housekeeping gene control. Images analyses were performed with Quantity One Software.

\section{Statistical analysis}

Data were expressed as mean \pm standard error (SE). All statistical analyses were performed with SPSS 11.0 software. analysis of variance (ANOVA) analysis was used, and a $P$-value $<0.05$ was considered statistically significant.

\section{Results}

\section{Syntheses of PEG-PEI copolymers}

PEG-PEI was synthesized through the conjugation of PEGNHS to PEI. 1H NMR analysis of the dialyzed PEI-PEG revealed prominent chemical shift of protons from $\mathrm{PEG}$ $\left(-\mathrm{OCH}_{2} \mathrm{CH}_{2}-, 3.65 \mathrm{ppm} ; \mathrm{CH}_{3} \mathrm{O}-, 3.38 \mathrm{ppm}\right)$ and PEI (- $\left.\mathrm{CH}_{2} \mathrm{CH}_{2} \mathrm{NH}-, 2.5-2.9 \mathrm{ppm}\right)$, indicating that PEG chains were successfully grafted onto PEI chains. PEG grafting density of PEI was 9.9 as characterized by $1 \mathrm{H}$ NMR (Figure 1).
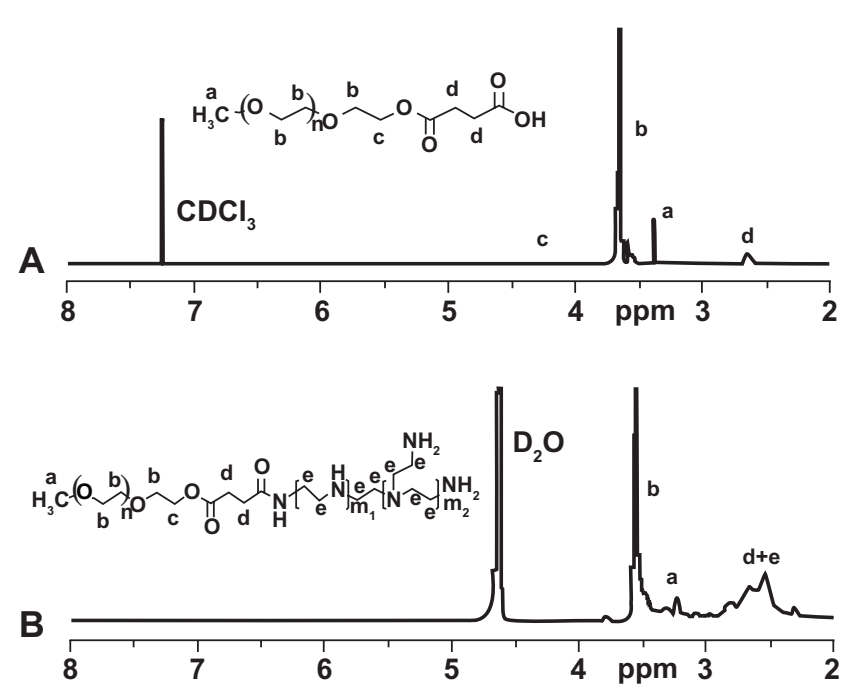

Figure I IH-NMR spectra of mPEG-COOH (A) and mPEG-PEI (B). Abbreviation: PEG-PEI, polyethylene glycol-polyethyleneimine.
Table I The particle size and zeta potential of PEG-PEI/siRNA complexes

\begin{tabular}{lllll}
\hline N/P ratio & $\mathbf{5}$ & $\mathbf{1 0}$ & $\mathbf{1 5}$ & $\mathbf{3 0}$ \\
\hline Size $(\mathrm{nm})$ & $174.6 \pm 1.2$ & $267.7 \pm 1.8$ & $241.5 \pm 3.8$ & $192.1 \pm 2.8$ \\
Zeta & $-5.76 \pm \mathrm{I} . \mathrm{I}$ & $3.01 \pm 0.1$ & $16.0 \pm 1.5$ & $26.9 \pm 0.8$ \\
potential $(\mathrm{mV})$ & & & & \\
\hline
\end{tabular}

Abbreviation: PEG-PEI, polyethylene glycol-polyethyleneimine.

\section{Size and zeta potential of PEG-PEI/siRNA complexes}

The size and zeta potential of PEG-PEI/siRNA complexes varied at different $\mathrm{N} / \mathrm{P}$ ratios; N/P ratios from 5 to 30 are shown in Table 1. At N/P 5, the size of the nanoparticle was approximately $174.6 \pm 1.2 \mathrm{~nm}$. The size reached to $267.7 \pm$ $1.8 \mathrm{~nm}$ at N/P 10 , then decreased gradually to $192.1 \pm 2.8 \mathrm{~nm}$ at N/P 30. The size distribution of PEG-PEI/siRNA at N/P 15 is shown in Figure 2; the size distribution of the complexes was between 180 and $242 \mathrm{~nm}$ whereas the remaining particles over $800 \mathrm{~nm}$ might be not complex well. The zeta potential was negative at N/P 5 and positive at N/P 10, increasing linearly as the ratio of PEG-PEI increased.

\section{Scanning electron microscope}

PEG-PEI/siRNA nanoparticles were well-complexed at N/P 15; therefore these particles were observed under SEM. The nanoparticles appeared spherical, uniform in size, and well-dispersed. The size of the nanoparticles at N/P 15 was approximately $240 \mathrm{~nm}$, which was similar to that measured with the Zeta-Plus instrument (Figure 3).

\section{Gel retardation assay}

The positive charges of PEG-PEI can neutralize the negative charges of the phosphate groups within the siRNA backbone,

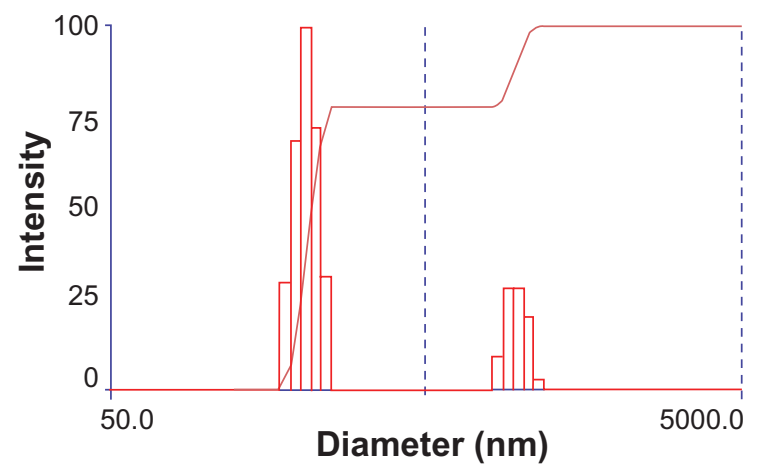

Figure 2 Size distribution of PEG-PEI nanoparticles at N/P 15 measured by Zeta Plus instrument.

Notes: The size distribution of two parts in the graph was respectively $180-242 \mathrm{~nm}$, 845-II $30 \mathrm{~nm}$.

Abbreviation: PEG-PEI, polyethylene glycol-polyethyleneimine. 


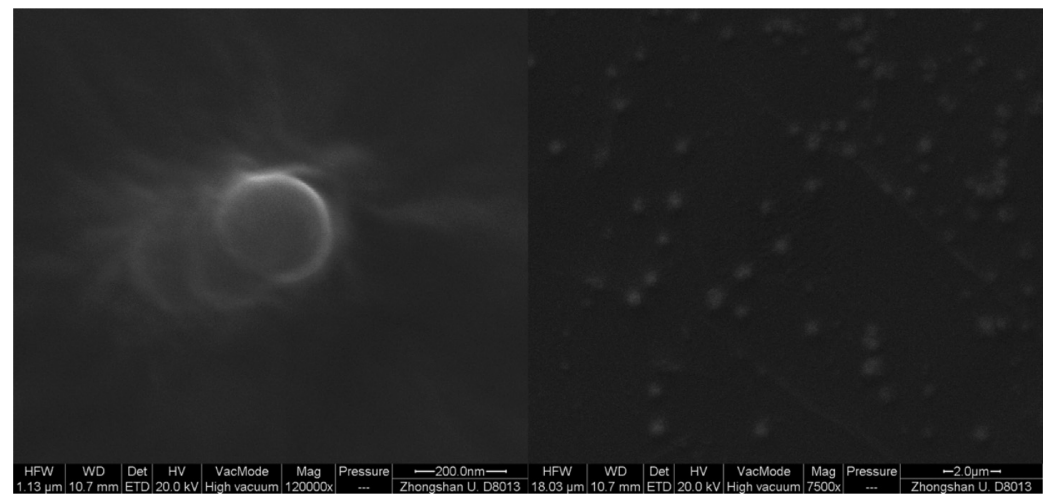

Figure 3 Scanning electron microscopy (SEM) images of PEG-PEI/siRNA nanoparticles at N/P I5 (I20000× and 7500× respectively).

Abbreviations: PEG-PEI, polyethylene glycol-polyethyleneimine; N/P, charge ratio between amino groups of PEG-PEI and phosphate groups of siRNA.

thus retarding the siRNA mobility (Figure 4). Naked siRNA was used as the control group. siRNA bands decreased with increasing N/P ratios (from 2.5 to 30 ). When the N/P was 10 , the band remained bright and completely disappeared when the N/P was 30 , indicating that the negatively charged siRNA could be neutralized entirely at this $\mathrm{N} / \mathrm{P}$ value.

\section{Cell viability analysis}

SGC7901 cells were incubated at different concentrations of PEG-PEI and PEI for 48 hours, and the cell viability was measured using the MTT assay (Figure 5A). Cells incubated with pure media, without treatment, were considered as controls, and the cell viability values was set at $100 \%$. Cytotoxicity of PEG-PEI was lower than that observed with PEI at the same concentrations $(P<0.05)$. When the concentrations were over $10 \mu \mathrm{g} / \mathrm{mL}$, increased toxicity was detected with PEG-PEI and PEI. As shown in Figure 5B, the cell viability in the presence of PEG-PEI/siRNA complexes at different

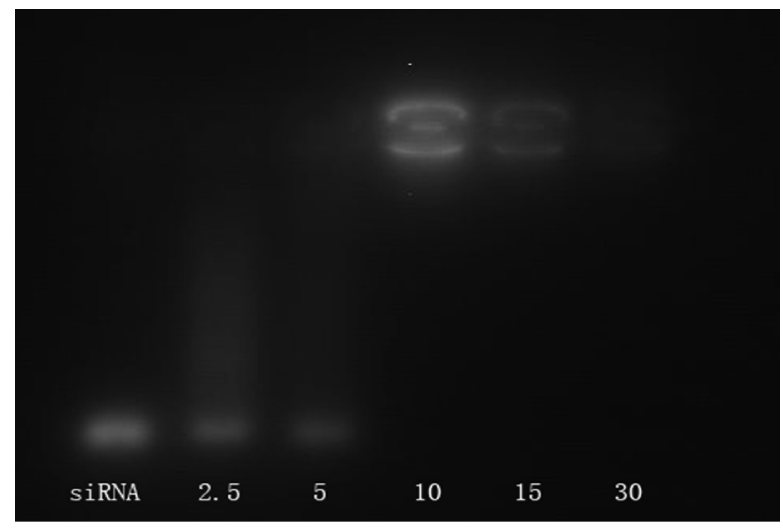

Figure $4 \mathrm{Gel}$ electrophoresis of PEG-PEI/siRNA complexes at different N/P ratios. Notes: Lanel: naked siRNA; Lane 2: PEG-PEI/siRNA at N/P 2.5; Lane 3: PEG-PEI/ siRNA at N/P 5; Lane 4: PEG-PEI/siRNA at N/P 10; Lane 5: PEG-PEl/siRNA at N/P 15; Lane 6: PEG-PEI/siRNA at N/P 30.

Abbreviations: PEG-PEI, polyethylene glycol-polyethyleneimine; N/P, charge ratio between amino groups of PEG-PEI and phosphate groups of siRNA.
$\mathrm{N} / \mathrm{P}$ ratios was tested after transfection for 48 hours. PEI $25 \mathrm{kDa} /$ siRNA and Lipofectamine 2000/siRNA complexes were used as controls. The cytotoxicity of PEG-PEI/siRNA complexes increased with higher N/P ratios. When the N/P

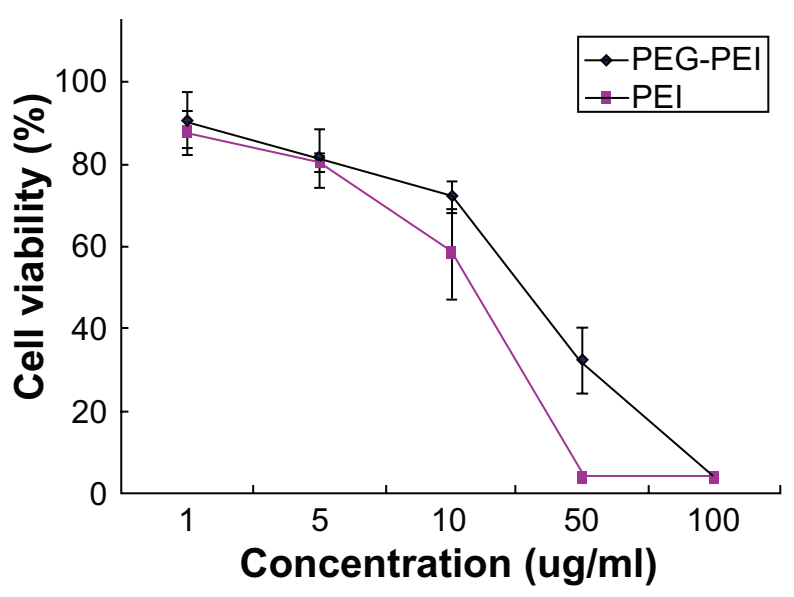

Figure 5a Cytotoxicity induced by the polymers of a variety of concentrations in SGC790I cells (mean $\pm \mathrm{SD}, \mathrm{n}=3, P<0.05$ ).

Abbreviations: $\mathrm{PEG}$, polyethylene glycol; $\mathrm{PEI}$, polyethyleneimine.

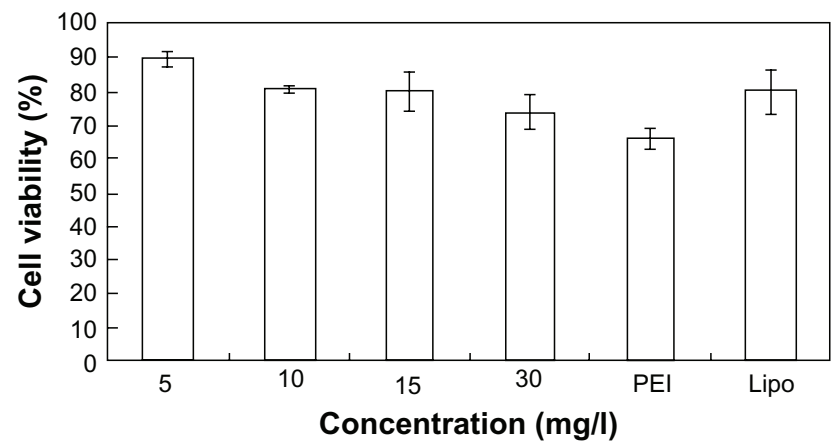

Figure 5b Cytotoxicity induced by PEG-PEl/siRNA complexes at different N/P ratios, PEI/siRNA and lipo2000/siRNA complexes after transfection into SGC790I cells (mean $\pm \mathrm{SD}, \mathrm{n}=3$ ).

Abbreviations: PEG-PEI, polyethylene glycol-polyethyleneimine; N/P, charge ratio between amino groups of PEG-PEI and phosphate groups of siRNA. 

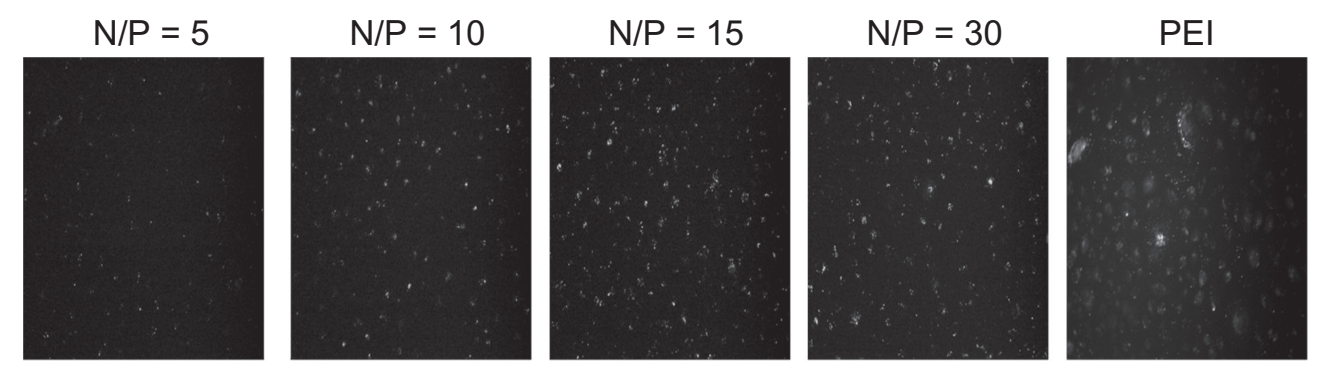

Lipo2000
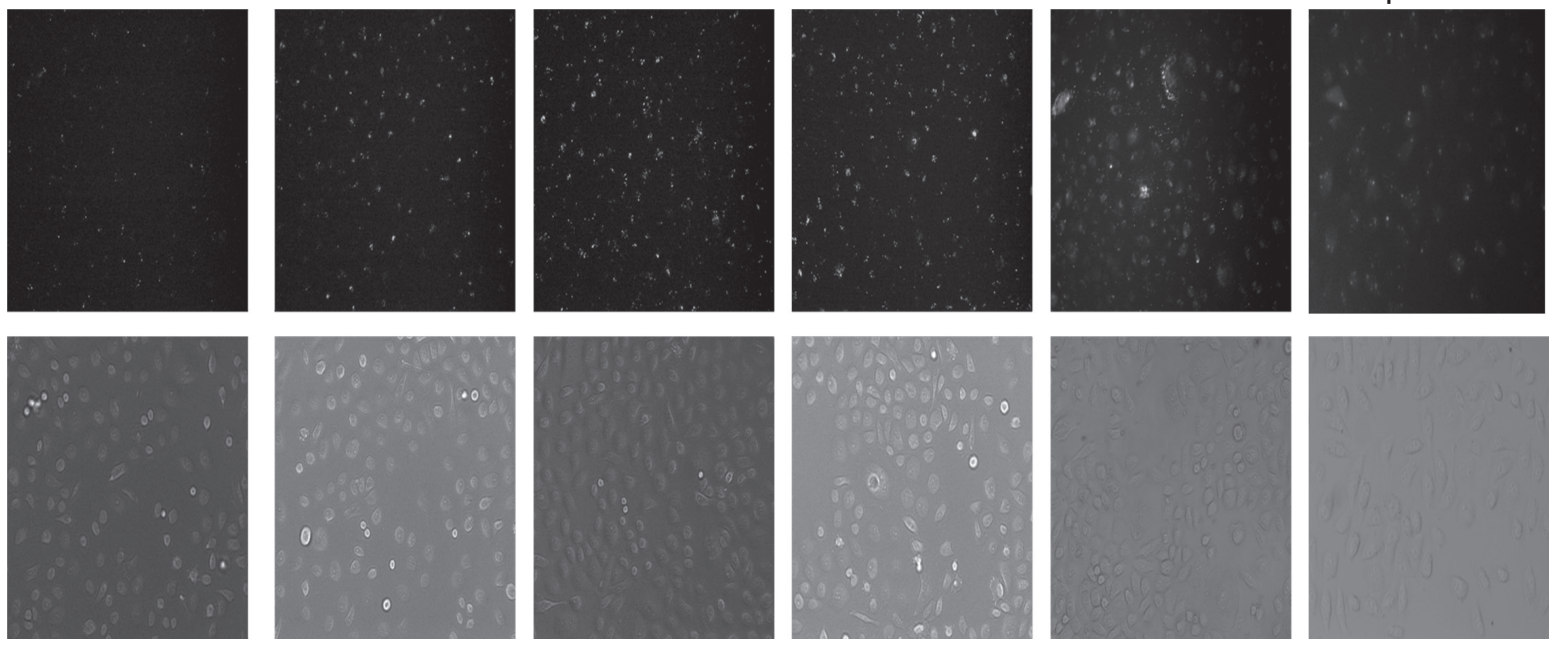

Figure 6a Fluorescence microscope images of SGC-790I cells transfected with PEG-PEI/siRNA complexes at N/P = 5, I0, I5, 30, PEI (25 KD) and Lipo2000, respectively. Abbreviations: PEG-PEl, polyethylene glycol-polyethyleneimine; N/P, charge ratio between amino groups of PEG-PEI and phosphate groups of siRNA.

ratio using PEG-PEI/siRNA reached 15 , the cell viability was $80.4 \% \pm 5.6 \%$, which was similar to that observed with Lipofectamine 2000/siRNA. When the N/P ratio was 30, the cell viability of cells transfected with PEG-PEI/siRNA was $73.8 \% \pm 4.8 \%$, which was higher than that of PEI/siRNA.

\section{In vitro transfection assay}

FAM-(carboxyfluorescein)-labeled siRNA was used in formation of the complexes, and transfection efficiency of PEG-PEI/siRNA complexes was evaluated with a fluorescence microscope and flow cytometry in SGC7901 cells. Lipofectamine 2000 and PEI ( $25 \mathrm{kDa}$ ) were used as positive controls. The transfection efficiencies appeared different at various N/P ratios (Figures $6 \mathrm{~A}$ and $6 \mathrm{~B}$ ); higher N/P ratios resulted in increased transfection efficiencies. For example, at N/P 5, the fluorescence intensity was weakest, increasing from N/P 5 to 30. Lipofectamine 2000 and PEI ( $25 \mathrm{kDa})$ also displayed high transfection efficiencies. The transfection efficiency of each group was evaluated in three independent experiments using flow cytometry. As shown in Figure 6B, the transfection efficiencies of PEG-PEI/siRNA at N/P 15 and 30 were $75.5 \% \pm 2.4 \%$ and $75.6 \% \pm 9.2 \%$, respectively. Although there was a minor increase, the transfection efficiencies of PEG-PEI/siRNA at N/P 30 were higher than that of Lipofectamine 2000/siRNA and PEI/siRNA.

\section{The gene silencing effect of CD44v6 siRNA}

PEG-PEI/siRNA at N/P 15 was chosen for transfection of the SGC7901 cells. The ability of siRNA to reduce CD44v6

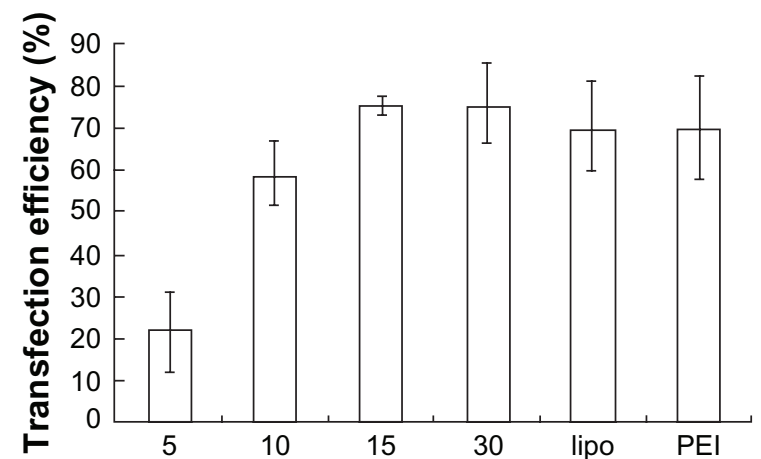

Figure 6b The transfection efficiencies of PEG-PEI/siRNA at different N/P ratios $(\mathrm{N} / \mathrm{P}=5,10,15,30)$, lipo2000 and PEI $(25 \mathrm{KD})$ measured by flow cytometry (mean \pm $\mathrm{SD}, \mathrm{n}=3)$.

Abbreviations: PEG-PEI, polyethylene glycol-polyethyleneimine; N/P, charge ratio between amino groups of PEG-PEI and phosphate groups of siRNA; SD, standard deviation.

mRNA and protein expression was analyzed using RT-PCR and Western blot analysis, respectively. Expression of CD44v6 was down-regulated by siRNA targeted to CD44v6 (Figure 7), indicating that siRNA-CD44v6 could suppress the expression of CD44v6 expression at the mRNA level while the negative control siRNA had no obvious effect on CD44v6 expression. The gene silencing effect of siRNA was further confirmed by Western blot analysis (Figure 8). The expression of CD44v6 protein was decreased using siRNACD44v6 to $59.7 \% \pm 3 \%$.

\section{Discussion}

RNAi-mediated silencing of important oncogenic regulators plays a major role in cancer therapy. For efficient cancer 


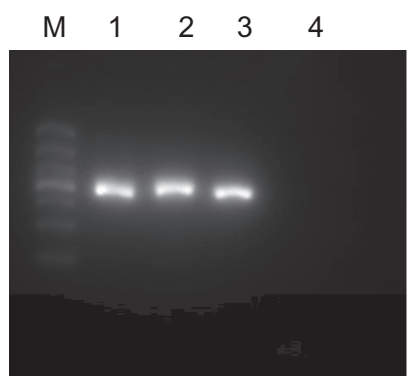

1. GADPH

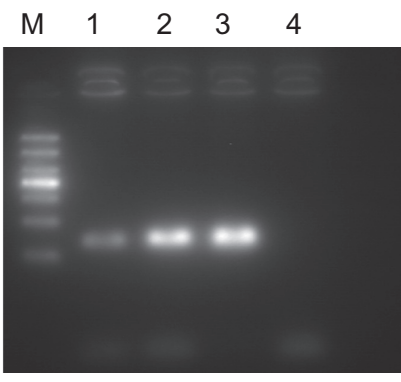

2. $\mathrm{CD} 44 \mathrm{v} 6$

Figure 7 The result of RT-PCR analysis after transfection with PEG-PEI/siRNA. Notes: M: marker. From the bottom: 100 bp, 200 bp, 300 bp, 400 bp, 500 bp, 700 bp 1000 bp. Lane I: siRNA-CD44v6/PEG-PEI complexes group. Lane 2: negative control siRNA/PEG-PEl complexes group. Lane 3:no treatment group. Lane 4: negative control group without RNA template. GADPH was used as a control.

Abbreviations: PEG-PEl, polyethylene glycol-polyethyleneimine; N/P, charge ratio between amino groups of PEG-PEI and phosphate groups of siRNA; GADPH, glyceraldehyde 3-phosphate dehydrogenase.

therapy, siRNA should be stable and efficiently delivered into the target tissue and easily taken up by the cancer cells. ${ }^{23}$ In this study, PEG (2 kDa)-PEI ( $25 \mathrm{kDa})$ was synthesized to form nanometer-sized complexes with siRNA. Reports have shown that adequate size and positive potential are essential for enhanced delivery of nanoparticles into cells. The size and potential of the complexes below N/P 10 were not suitable for cell transfection (Table 1) because PEG-PEI and siRNA could not form a complex of adequate size and positive potential.

Agarose electrophoresis was used to evaluate siRNAcopolymer affinity. When PEG-PEI/siRNA complexes formed, the negatively charged siRNA could be neutralized by the positively charged polycations. Thus, insertion of colloidal gold into the siRNA was impeded. With N/P ratios increasing, siRNA was complexed more effectively with the polycations; the insertion of colloidal gold into siRNA double-strands was blocked to a higher extent and consequently the brightness of the siRNA bands weakened. ${ }^{24}$ In this study, at N/P 10, the complete neutralization of the siRNA was observed. At N/P 30, siRNA was entirely wrapped by PEG-PEI, producing a fluorescence quenching phenomenon.

1

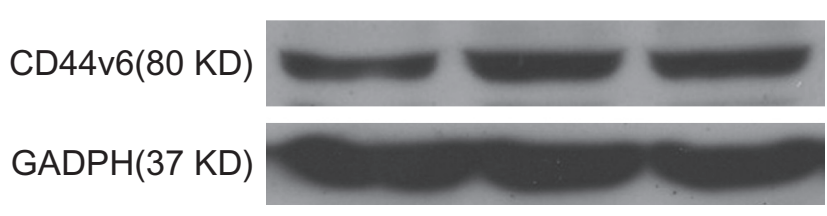

Figure 8 The result of Western blot analysis after transfection with PEG-PEI/ siRNA.

Notes: Lane I: siRNA-CD44v6/PEG-PEl complexes group. Lane 2: negative control siRNA/PEG-PEI complexes group. Lane 3: no treatment group.

Abbreviations: PEG-PEI, polyethylene glycol-polyethyleneimine; GADPH, glyceraldehyde 3-phosphate dehydrogenase.
Therefore, it could be considered that siRNA was completely complexed with PEG-PEI at N/P ratios $\geq 10$. Interaction of DNA or siRNA with cationic polymers could protect the condensed DNA or siRNA from enzymatic degradation, ${ }^{25,26}$ making it more effective for cellular transfection.

Increased transfection efficiency of PEG-PEI/siRNA was observed at higher N/P ratios, reaching the highest transfection efficiency at N/P 15 . No major increase appeared at N/P 30 possibly because over-condensation at high N/P values is not favorable for intracellular release of nucleic acids. ${ }^{27,28}$

Cytotoxicity of the PEG-PEI/siRNA was also analyzed using the MTT assay. Cytotoxicity induced by PEG-PEI was obviously lower than PEI, which suggests that PEG reduced the toxicity of the heavily cationic PEI portion of copolymer structure. ${ }^{13}$ When the concentration of PEG-PEI in the culture medium was less than $10 \mu \mathrm{g} / \mathrm{mL}$, the biosafety of this delivery agent was improved. As a non-viral delivery system, Lipofectamine 2000 is widely used in studies analyzing RNAi. However, some reports have indicated that Lipofectamine 2000 has a high toxicity. ${ }^{29,30}$ In this study, when N/P values were below 15 , the cell toxicity of PEG-PEI/siRNA was lower than that of Lipofectamine 2000/siRNA, indicating that PEG-PEI may be a promising non-viral gene delivery carrier with higher transfection efficiency and lower cytotoxicity.

Considering the high transfection efficiency and low cytotoxicity, PEG-PEI/siRNA at N/P 15 this was chosen to transfect SGC7901 cells. RT-PCR and Western blot analyses indicated effective gene silencing of CD44v6 expression in SGC7901 cells when PEG-PEI served as a carrier. The results showed that PEG modified-PEI (25 kDa) delivered siRNA into SGC7901 cells and released them effectively.

Compared with PEI (25 kDa) and Lipofectamine 2000, PEG-PEI has more advantages, such as increased transfection efficiency and improved cell viability. However, this carrier may be further improved. We are dedicated to research on the linkage of single-chain antibody and PEG-PEI $(25 \mathrm{kDa})$. The study will improve the transfection efficiency and minimize the damage of siRNA to normal gastric cells.

\section{Conclusion}

In this study, we developed a nano-sized polymeric system for delivering siRNA to inhibit the expression of CD44v6 in gastric cancer cells in vitro. Compared with Lipofectamine 2000 or PEI $(25 \mathrm{kDa})$, the copolymer had advantages such as low cytotoxicity, high transfection efficiency, low cost, with easy adjustment of its structure. Thus, PEG-PEI might be a promising gene carrier for siRNA in gene therapy of gastric cancer. Moreover, this study provided a foundation 
for related research on PEG-PEI/siRNA nanoparticles in vivo and gene targeted delivery.

\section{Acknowledgements}

This work was supported by the Natural Science Foundation of China (Grant Number 30670951), the Natural Science Foundation of Guangdong province (Grant Number 6021322).

\section{Disclosures}

The authors report there are no conflicts of interest relevant to this research.

\section{References}

1. Yamaguchi A, Goi T, Yu J, et al. Expression of CD44v6 in advanced gastric cancer and its relationship to hematogenous metastasis and long-term prognosis. J Surg Onc. 2002;79:230-235.

2. Bourguignon LY, Zhu D, Zhu H. CD44 isoform-cytoskeleton interaction in oncogenic signaling and tumor progression. Frontiers in Bioscience. 1998;3:637-649.

3. Yamamichi K, Uehara Y, Kitamura N, et al. Incresed expression of CD44v6 mRNA significantly correlates with distant metastasis and poor prognosis in gastric cancer. Int J Cancer. 1998;79:256-262.

4. Kurozumi K, Nishida T, Nakao K, et al. Expression of CD44 variant 6 and lymphatic invasion: importance to lymph node metastasis in gastric cancer. World J Surg. 1998;22:853-857.

5. Fuchs F, Boutros M. Cellular phenotyping by RNAi. Brief Funct Genomic Proteomic. 2006;5(1):52-56.

6. Cullen LM, Arndt GM. Genome-wide screening for gene function using RNAi in mammalian cells. Immunol Cell Bio. 2005;83:217-223.

7. Takeshita F, Ochiya T. Therapeutic potential of RNA interference against cancer. Cancer Sci. 2006;7(8):689-696.

8. Bitko V, Musiyenko A, Shulyayeva O, Barik S. Inhibition of respiratory viruses by nasally administered siRNA. Nat Med. 2005;11(1):50-55.

9. Kim SH, Jeong JH, Lee SH, et al. PEG conjugated VEGF siRNA for antiangiogenic gene therapy. J Controlled Release. 2006;116:123-129.

10. Werth S, Urban-Klein B, Dai L, et al. A low molecular weight fraction of polyethyleneimine (PEI) displays increased transfection efficiency of DNA and siRNA in fresh or lyophilized complexes. J Controlled Release. 2006;112:257-270.

11. Read ML, Logan A, Seymour LW. Barriers to gene delivery using synthetic vectors. Adv Genet. 2005;52:19-46.

12. Andersen MØ, Howard KA, Paludan SR, et al. Delivery of siRNA from lyophilized polymeric surfaces. Biomaterials. 2008;29:506-512.

13. Sutton D, Kim S, Shuai X, et al. Efficient suppression of secretory clusterin levels by polymer-siRNA nanocomplexes enhances ionizing radiation lethality in human MCF-7 breast cancer cell in vitro. Int $J$ Nanomedicine. 2006;1(2):155-162.

14. Chen G, Chen W, Wu Z, et al. MRI-visible polymeric vector bearing CD3 single chain antibody for gene delivery to T cells for immunosuppression. Biomaterials. 2009;30:1962-1970.
15. Nguyen HK, LemieuxP, Vinogradov SV, et al. Evaluation of polyetherpolyethyleneimine graft copolymers as gene transfer agents. Gene Ther. 2000;7:126-138.

16. Liang B, He ML, Xiao ZP, et al. Synthesis and characterization of folate-PEG-grafted-hyperbranched-PEI for tumor-targeted gene delivery. Biochem Biophys Res Commun. 2008;367:874-880.

17. Fischer D, Osburg B, Petersen H, Kissel T, Bickel U. Effect of poly (ethyleneimine) molecular weight and pegylation on organ distribution and pharmacokinetics of polyplexes with oligodeoxynucleotides in mice. Drug Metab Dispos. 2004;32:983-992.

18. Fischer D, von Harpe A, Kunath $\mathrm{K}$, et al. Copolymers of ethyleneimine and $\mathrm{N}$-(2-hydroxyethyl)-ethyleneimine as tools to study effects of polymer structure on physicochemical and biological properties of DNA complexes. Bioconjug Chem. 2002;13:1124-1133.

19. Neu M, Germershaus O, Behe M, et al. Bioreversibly crosslinked polyplexes of PEI and high molecular weight PEG show extended circulation times in vivo. J Control Release. 2007;124(1-2):69-80.

20. Tang GP, Zeng JM, Gao SJ, et al. Polyethylene glycol modified polyethylenimine for improved CNS gene transfer: effects of PEGylation extent. Biomaterials. 2003;24(13):2351-2362.

21. Liu X, Howard KA, Dong M, et al. The influence of polymeric properties on chitosan/siRNA nanoparticle formulation and gene silencing. Biomaterials. 2007;28(6):1280-1288.

22. Shuai X, Merdan T, Unger F, et al. Novel biodegradable ternary copolymers hy-PEI-g-PCL-b-PEG: synthesis, characterization, and potential as efficient nonviral gene delivery vectors. Macromolecules. 2003;36: 5751-5759.

23. Sun Hwa Kim, Ji Hoon Jeong, Soo Hyeon Lee, et al. Local and systemic delivery of VEGF siRNA using polyelectrolyte complex micelles for effective treatment of cancer. J Controlled Release. 2008;129(2): 107-116.

24. Kainthan RK, Gnanamani M, Ganguli M, et al. Blood compatibility of novel water soluble hyperbranched polyglycerol-based multivalent cationic and their interaction with DNA. Biomaterials. 2006;27(31): $5377-5390$.

25. Harada A, Togawa H, Kataoka K. Physicochemical properties and nuclease resistance of antisense-oligonucleotides entrapped in the core of polyion complex micelles composed of poly(ethylene glycol)-poly (L-lysine) block copolymers. Eur J Pharm Sci. 2001;13:35-42.

26. Jeong JH, Kim SW, Park TG. Novel intracellular delivery system of antisense oligonucleotide by self assembled hybrid micelles composed of DNA/PEG conjugate and cationic fusogenic peptide. Bioconjug Chem. 2003;14:473-479.

27. Ogris M, Steinlein P, Kursa M, et al. The size of DNA/transferrin-PEI complexes is an important factor for gene expression in cultured cells. Gene Ther. 1998;5(10):1425-1433.

28. Lungwitz U, Breunig M, Blunk T, et al. Polyethylenimine-based non-viral gene delivery systems. Eur J Pharm Biopharm. 2005;60(2): 247-266.

29. Jere D, Yoo MK, Arote R, et al. Poly (amino ester) composed of poly (ethylene glycol) and aminosilane prepared by combinatorial chemistry as a gene carrier. Pharm Res. 2008;25(4):875-885.

30. Corsi K, Chellat F, Yahia L, et al. Mesenchymal stem cells, MG63 and HEK293 transfection using chitosan-DNA nanoparticles. Biomaterials. 2003;24(7):1255-1264.
International Journal of Nanomedicine

\section{Publish your work in this journal}

The International Journal of Nanomedicine is an international, peerreviewed journal focusing on the application of nanotechnology in diagnostics, therapeutics, and drug delivery systems throughout the biomedical field. This journal is indexed on PubMed Central, MedLine, CAS, SciSearch $\AA$, Current Contents $₫ /$ Clinical Medicine,

\section{Dovepress}

Journal Citation Reports/Science Edition, EMBase, Scopus and the Elsevier Bibliographic databases. The manuscript management system is completely online and includes a very quick and fair peer-review system, which is all easy to use. Visit http://www.dovepress.com/ testimonials.php to read real quotes from published authors. 